\title{
Grado de incumplimiento terapéutico en función del nivel de alfabetización en salud en pacientes en hemodiálisis
}

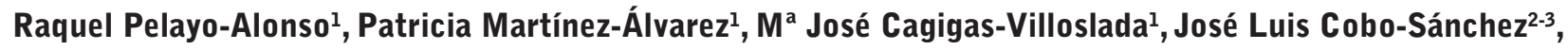 \\ Clara M ${ }^{\mathrm{a}}$ Palacio-Cornejo \\ ${ }^{1}$ Servicio de Nefrología. Hospital Universitario Marqués de Valdecilla. Santander. España \\ ${ }^{2}$ Subdirección de Cuidados. Servicio Cántabro de Salud. Santander. España \\ ${ }^{3}$ Escuela Universitaria de Enfermería Clínica Mompía. Universidad Católica de Ávila. Mompía. España
}

\begin{abstract}
Como citar este artículo:
Pelayo-Alonso R, Martínez-Álvarez P, Cagigas-Villoslada MJ, Cobo-Sánchez JL, Palacio-Cornejo CM. Grado de incumplimiento terapéutico en función del nivel de alfabetización en salud en pacientes en hemodiálisis

Enferm Nefrol. 2021 Abr-Jun;24(2):175-82
\end{abstract}

\section{Resumen}

Introducción: El paciente en hemodiálisis está sometido a un régimen terapéutico cuya adherencia contribuye a disminuir la aparición de complicaciones asociadas. EI incumplimiento terapéutico es un problema prevalente en la práctica clínica. Bajos niveles de alfabetización en salud se relacionan con falta de autocuidados, errores en la toma de medicaciones y dificultad para entender instrucciones, lo que trae consigo una mayor tasa de incumplimiento terapéutico.

Objetivo: Conocer el grado de incumplimiento terapéutico en función del grado de alfabetización en salud de los pacientes sometidos a HD y valorar otros posibles factores implicados.

Material y Método: Estudio prospectivo en 35 pacientes en programa de hemodiálisis. La alfabetización en salud se valoró con el cuestionario Health Literacy Survey European Union y el grado de incumplimiento terapéutico mediante la ganancia de peso interdialítica e indicadores analíticos, según recomendaciones de guías clínicas.

Resultados: Los pacientes con nivel de alfabetización adecuada ( $\geq 34$ puntos) presentaron menor incumpli-

\section{Correspondencia:}

Raquel Pelayo Alonso

Email: sanesteban6@gmail.com miento terapéutico que pacientes con alfabetización inadecuada en relación con la ganancia de peso interdialítica $(78 \%$ vs $70 \%)$, potasio $(67 \%$ vs $56 \%)$, albúmina ( $83 \%$ vs $72 \%$ ) pero mayor IT con el fósforo $(83,33 \%$ vs $51,44 \%)-p<0,05$ en todos los casos-.

Conclusiones: Los pacientes con mayor nivel de alfabetización en salud tienen un menor grado de incumplimiento terapéutico, a excepción del fósforo, que es el parámetro con peor tasa de adherencia.

PALABRAS CLAVE: hemodiálisis; alfabetización en salud; adherencia al tratamiento; incumplimiento terapéutico.

\section{Degree of non-compliance with treatment as a function of health literacy level in haemodialysis patients}

\section{Summary}

Introduction: Haemodialysis patients are subject to a therapeutic regimen whose adherence helps to reduce the occurrence of associated complications. Non-compliance is a prevalent problem in clinical practice. Low levels of health literacy are associated with lack of self-care, errors 
in taking medications and difficulty in understanding instructions, leading to a higher rate of non-compliance.

Objective: To determine the level of non-compliance with treatment according to the level of health literacy of haemodialysis patients and to assess other possible factors involved.

Material and Method: Prospective study of 35 haemodialysis patients. Health literacy was assessed using the Health Literacy Survey European Union questionnaire and the degree of therapeutic non-compliance was assessed using interdialytic weight gain and analytical indicators, according to the recommendations of clinical guidelines.

Results: Patients with adequate literacy ( $\geq 34$ points) had lower non-compliance than patients with inadequate literacy in relation to interdialytic weight gain $(78 \%$ vs $70 \%)$, potassium $(67 \%$ vs $56 \%$ ), albumin $(83 \%$ vs $72 \%)$; but higher non-compliance with phosphorus $(83.33 \%$ vs $51.44 \%)-p<0.05$ in all cases.

Conclusions: Patients with a higher level of health literacy have a lower degree of non-compliance, except for phosphorus, which is the parameter with the worst adherence rate.

KEYWORDS: haemodialysis; health literacy; treatment adherence; non-compliance.

\section{Introducción}

El paciente en hemodiálisis (HD) está sometido a un régimen terapéutico que incluye varias modificaciones importantes de su estilo de vida ${ }^{1}$. La adherencia a este régimen terapéutico contribuye a disminuir la aparición de complicaciones asociadas y, por lo tanto, a mejorar la morbimortalidad ${ }^{2}$. El incumplimiento terapéutico (IT) o falta de adherencia al tratamiento es un problema prevalente y relevante en la práctica clínica, frecuentemente en enfermedades crónicas con prevalencias del 30-60\% según la patología 3 .

La Organización Mundial de la Salud (OMS) definió la adherencia al tratamiento prolongado como "el grado en que el comportamiento de una persona (tomar el medicamento, seguir un régimen alimentario y ejecutar cambios del modo de vida) se corresponde con las recomendaciones acordadas por un prestador de asistencia sanitaria"'3. Para favorecer el cumplimiento terapéutico, los profesionales sanitarios debemos proporcionar a los pacientes información apropiada que les permita aumentar su conocimiento, habilidades y capacidades para que tomen decisiones autónomas y mejoren sus autocuidados $^{2}$, teniendo en cuenta siempre su nivel educativo y su grado de alfabetización en salud $(A e S)^{4,5}$, definida según la OMS, como "las habilidades sociales y cognitivas que determinan el nivel de motivación y la capacidad de una persona para acceder, entender, y utilizar la información de forma que le permita promover y mantener una buena salud"6. Unos bajos niveles de AeS se relacionan con falta de actividad de autocuidados, errores en la toma de medicaciones y dificultad para entender instrucciones ${ }^{6,7}$, lo que trae consigo una mayor tasa de IT.

El objetivo de este estudio fue conocer el grado de incumplimiento terapéutico en función del grado de AeS de los pacientes sometidos a HD y valorar otros posibles factores implicados.

\section{Material y Método}

Se realizó un estudio descriptivo prospectivo en 35 pacientes mayores de 18 años sometidos a HD crónica durante más de 3 meses en la Unidad de Hemodiálisis del Hospital Universitario Marqués de Valdecilla (HUMV). Se excluyeron a aquellos pacientes que en el momento del estudio presentaron una situación clínica que impidiese la cumplimentación del cuestionario, pacientes con deterioro cognitivo, mala comprensión del idioma y la negativa del paciente a participar en el estudio tras recibir la información pertinente. Todos los pacientes firmaron el consentimiento informado antes del inicio de la recogida de datos.

Para valorar el nivel de AeS se utilizó el cuestionario Health Literacy Survey European Union (HLS$E U-Q)^{8}$ en su versión española validada, cuestionario que consta de 47 ítems sobre situaciones relacionadas sobre la gestión de la información, asistencia sanitaria, prevención de la enfermedad y promoción de la salud. El cuestionario fue autocumplimentado por el paciente recibiendo ayuda del equipo investigador aquellos pacientes que así lo solicitaron. Cada ítem se responde en una escala tipo Likert de 4 puntos (de muy fácil a muy difícil). Se obtiene un índice general de AeS con una puntuación de 0-50 puntos; y 3 subíndices secundarios (Atención y cuidados de salud, Prevención de Enfermedades y Promoción de Salud), con el mismo rango de puntuación posible. Según la puntuación alcanzada en el índice general de AeS se establecen 4 niveles de 
AeS: inadecuada (0-25 puntos), problemática (26-33 puntos), suficiente (34-42 puntos) y excelente (42-50 puntos).

El IT se evaluó mediante la valoración de la ganancia de peso interdialítica (GPI) y cifras analíticas de potasio, albúmina, fósforo, proteínas y calcio sérico durante los 3 meses previos a la determinación del nivel de AeS, estableciéndose como parámetros aceptables cifras $<2300 \mathrm{~g}$ para la GPI -según norma interna de la Unidad se admite una GPI de $2000 \mathrm{~g}$ y $2500 \mathrm{~g}$ entre sesiones del fin de semana-; potasio $<5,5 \mathrm{mEq} / \mathrm{L}$; albúmina $\geq 3,5 \mathrm{mg} / \mathrm{dl}$; fósforo $<4,5 \mathrm{mg} / \mathrm{dl}$; proteínas $\geq 6 \mathrm{~g} / \mathrm{dl}$ y cifras de calcio de 8,4-9,5 mg/dl, según recomendaciones de las guías clínicas ${ }^{9}$ y del servicio de Bioquímica del HUMV. Se recogieron datos sociodemográficos y clínicos de la historia clínica del paciente: edad, sexo, índice de comorbilidad según índice de Charlson.

Para el análisis de los datos se utilizó el paquete estadístico IBM SPSS statistics versión 20 . Se realizó un análisis descriptivo, utilizando medidas de tendencia central para las variables cuantitativas, y frecuencias y porcentajes para las variables cualitativas. Para la inferencia estadística se utilizaron los test de la $T$ de student, $U$ de Mann-Whitney y ANOVA de un factor, considerándose un valor de $p<0,05$ como estadísticamente significativo. Para el análisis por edad se estableció como punto de corte la mediana de edad de la muestra a estudio. Para el análisis para el índice general y cada subíndice del HLS-EU-Q se establecieron 2 grupos: nivel adecuado con una puntuación $\geq 34$ puntos y nivel inadecuado con puntuaciones por debajo de 34 puntos.

\section{Resultados}

Se estudiaron 35 pacientes con una edad media de $65,29 \pm 16,70$ años (mediana 69 años) siendo un $57,1 \%$ hombres, con una comorbilidad media según el Índice de Charlson de 4,74 $\pm 1,4$ puntos (mediana 5 puntos).

El valor medio del índice general de AeS fue de $26,8 \pm 7,26$ puntos. Las puntuaciones obtenidas en los subíndices fueron: atención y cuidados de salud $27,9 \pm 8,16$ puntos, prevención de enfermedades $27,2 \pm 8,59$ puntos y promoción de la salud $24,6 \pm 7,44$ puntos. Según los niveles de AeS en función de la puntuación obtenida los pacientes con AeS adecuada ( $\geq 34$ puntos) alcanzaron un $17,14 \%, 51,43 \%$ inadecuada, 31,43\% problemática, $14,28 \%$ suficiente y $2,86 \%$ excelente.

Los datos referentes al grado de cumplimiento terapéutico se especifican en la Tabla 1. Los pacientes con un índice general de AeS adecuado, presentaron menor grado de IT que aquellos pacientes con niveles de AeS inadecuados, tanto para la GPI, potasio sérico y niveles de albúmina. Sin embargo, en el caso del fósforo sérico, aquellos pacientes con un mayor nivel de AeS presentan peor grado de IT ( $83 \%$ frente a $51 \% ; p=0,042)$. En la Tabla 2 se detallan los datos relativos al porcentaje de incumplimiento terapéutico según el nivel de AeS, tanto en función del índice general, como según los 3 subíndices que establece el cuestionario empleado.

En función del sexo, los hombres presentaron un mayor grado de cumplimiento terapéutico del nivel de fósforo sérico (hombres $55,42 \%$ vs mujeres $26,67 \%$; $p=0,023$ ). En la Tabla 3 se pueden ver los diferentes parámetros alcanzados según el sexo, así como su grado de cumplimiento.

Los datos de IT según la edad se detallan en la Tabla 4. Los pacientes mayores de 69 años (mediana de la muestra) mantienen un fósforo medio de $4,38 \pm 1,15$ $\mathrm{mg} / \mathrm{dl}$ frente a los $5,38 \pm 1,33 \mathrm{mg} / \mathrm{dl}$ que alcanzan los menores de 69 años $(p=0,041)$ que además presentan un grado de IT del $70 \%(p=0,055)$.

Tabla 1. Parámetros de cumplimiento terapéutico según indicador establecido.

\begin{tabular}{|l|c|c|c|c|c|}
\hline Parámetro (unidad) & Media & DE & Mínimo & Máximo & \% Incumplimiento indicador \\
\hline GPI (g) & 1790,76 & 711,5 & 379 & 3449 & $28,4 \%$ \\
\hline Potasio (mEq/L) & 5,4 & 0,9 & 2,8 & 7,1 & $42,4 \%$ \\
\hline Albúmina (g/dl) & 3,6 & 0,3 & 2,9 & 4,1 & $26,2 \%$ \\
\hline Proteínas (g/dl) & 6,3 & 0,5 & 5,1 & 7,4 & $24,7 \%$ \\
\hline Fósforo (mg/dl) & 4,7 & 1,3 & 3 & 8,6 & $56,9 \%$ \\
\hline Calcio (mg/dl) & 8,7 & 0,8 & 6,8 & 7,2 & $48,2 \%$ \\
\hline
\end{tabular}

GPI: ganancia de peso interdiálisis. Indicadores: GPI $<2300 \mathrm{~g}$, potasio $<5,5 \mathrm{mEq} / \mathrm{L}$; albúmina $\geq 3,5 \mathrm{mg} / \mathrm{dl}$; fósforo $<4,5 \mathrm{mg} / \mathrm{dl}$; proteínas $\geq 6 \mathrm{~g} / \mathrm{dl}$ y cifras de calcio de $8,4-9,5 \mathrm{mg} / \mathrm{dl}$. 
Tabla 2. Valores medios de los diferentes parámetros de cumplimiento terapéutico establecidos según el nivel de Alfabetización en Salud.

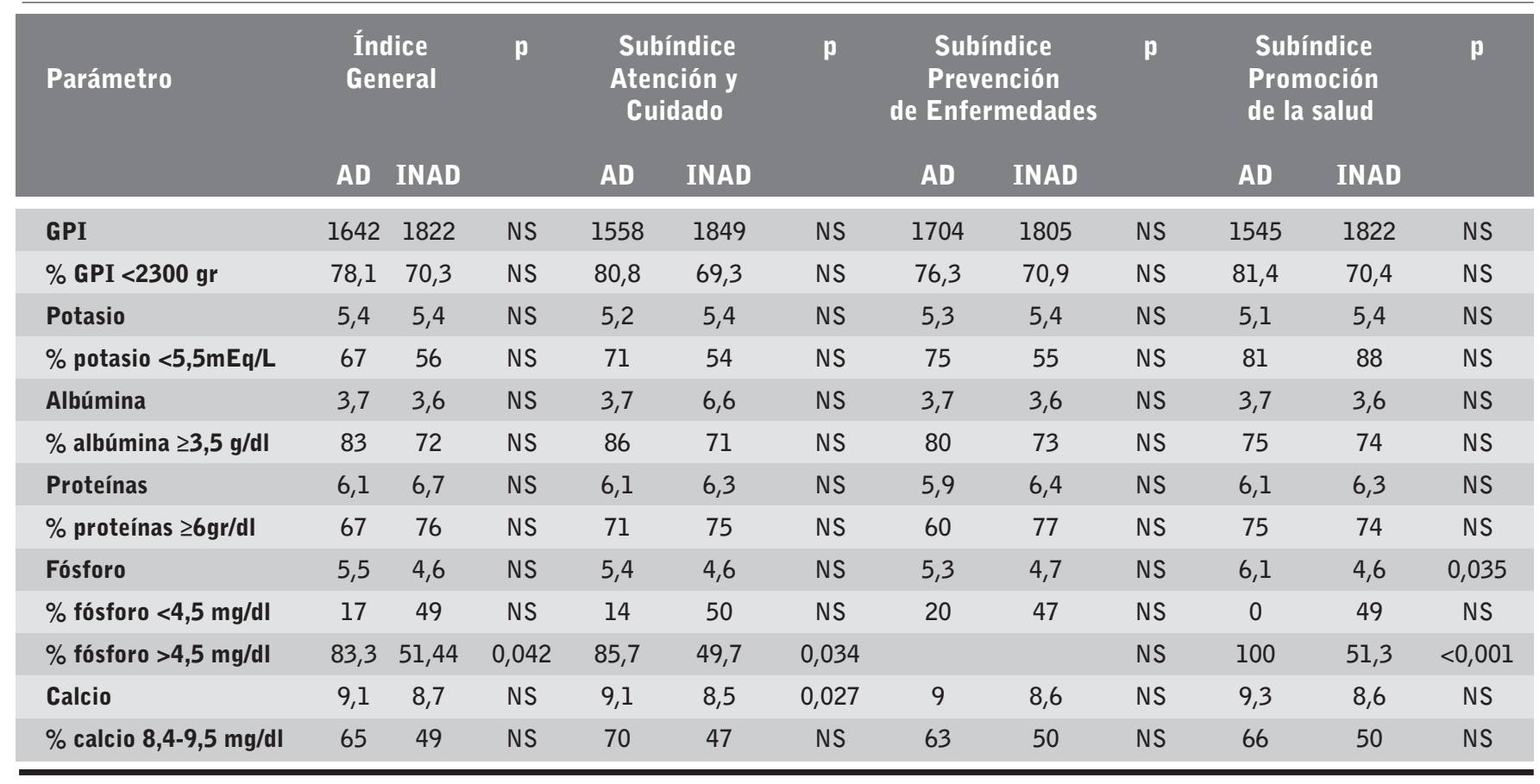

GPI: ganancia de peso interdiálisis. Indicadores: GPI $<2300 \mathrm{~g}$, potasio $<5,5 \mathrm{mEq} / \mathrm{L}$; albúmina $\geq 3,5 \mathrm{mg} / \mathrm{dl}$; fósforo $<4,5 \mathrm{mg} / \mathrm{dl} ;$ proteínas $\geq 6 \mathrm{~g} / \mathrm{dl}$ y cifras de calcio de $8,4-9,5 \mathrm{mg} / \mathrm{dl}$.

Tabla 3. Valores medios de los diferentes parámetros y del porcentaje de cumplimiento terapéutico establecidos según el sexo.

\begin{tabular}{|c|c|c|c|c|c|}
\hline \multirow[b]{2}{*}{ PARAMETRO } & \multicolumn{2}{|c|}{ HOMBRE } & \multicolumn{2}{|c|}{ MUJER } & \multirow[t]{2}{*}{$\mathbf{p}$} \\
\hline & Media & DE & Media & DE & \\
\hline Puntuación general AeS & 25,9 & 6,01 & 28,1 & 8,7 & NS \\
\hline GPI (g) & 1865 & 763,3 & 1615 & 758,7 & NS \\
\hline$\%$ GPI $<2300$ g & 67 & 26,8 & 77,8 & 23,9 & NS \\
\hline$\%$ GPI $\geq 2300 \mathrm{~g}$ & 33 & 20,8 & 22,2 & 23,9 & NS \\
\hline Potasio (mEq/L) & 5,4 & 1,1 & 5,3 & 0,6 & NS \\
\hline Número ocasiones $\mathrm{K}+<5,5 \mathrm{mEq} / \mathrm{L}$ & 1,9 & 1,6 & 2,3 & 1,5 & NS \\
\hline$\%$ Potasio $<5,5 \mathrm{mEq} / \mathrm{L}$ & 55 & 43,2 & 61,1 & 29,5 & NS \\
\hline$\%$ Potasio $\geq 5,5 \mathrm{mEq} / \mathrm{L}$ & 45 & 43,2 & 38,9 & 29,5 & NS \\
\hline Albúmina (g/dl) & 3,7 & 0,3 & 3,5 & 0,3 & NS \\
\hline$\%$ Albúmina $\geq 3,5 \mathrm{~g} / \mathrm{dl}$ & 84,2 & 32,2 & 60 & 42,2 & 0,045 \\
\hline Proteínas (g/dl) & 6,4 & 0,5 & 6,2 & 0,6 & NS \\
\hline$\%$ Proteínas $\geq 6 \mathrm{gr} / \mathrm{dl}$ & 77,9 & 39,4 & 69,4 & 40,8 & NS \\
\hline Fósforo (mg/dl) & 4,4 & 1,1 & 5,4 & 1,4 & 0,037 \\
\hline$\%$ fósforo $<4,5 \mathrm{mg} / \mathrm{dl}$ & 55,5 & 35,9 & 26,7 & 42,2 & 0,023 \\
\hline$\%$ fósforo $\geq 4,5 \mathrm{mg} / \mathrm{dl}$ & 44,6 & 38,9 & 73,3 & 42,2 & 0,023 \\
\hline Calcio (mg/dl) & 8,5 & 0,9 & 8,8 & 0,7 & NS \\
\hline$\%$ Calcio 8,4-9,5 mg/dl & 48,3 & 42,2 & 56,5 & 41,1 & NS \\
\hline
\end{tabular}

AeS: Alfabetización en Salud; GPI: Ganancia de peso interdialítica. NS: no significativo $(p>0,05)$. 
Tabla 4. Valores medios de los diferentes parámetros y del porcentaje de cumplimiento terapéutico establecidos según edad.

\begin{tabular}{|c|c|c|c|c|c|}
\hline \multirow[b]{2}{*}{ PARAMETRO } & \multicolumn{2}{|c|}{$\geq 69$ años } & \multicolumn{2}{|c|}{$<69$ años } & \multirow[t]{2}{*}{$\mathbf{p}$} \\
\hline & Media & DE & Media & DE & \\
\hline Índice general AeS & 25,1 & 6,3 & 28,5 & 7,9 & NS \\
\hline GPI (g) & 1536,1 & 683,6 & 1992,6 & 786,6 & NS \\
\hline$\%$ GPI $<2300 \mathrm{gr}$ & 79,1 & 22,4 & 63,8 & 27,5 & NS \\
\hline$\%$ GPI $\geq \mathbf{2 3 0 0 ~ g r}$ & 19,2 & 22,8 & 36,2 & 27,5 & 0,059 \\
\hline Potasio (mEq/L) & 5,3 & 0,8 & 5,4 & 1,1 & NS \\
\hline Número ocasiones potasio $<5,5 \mathrm{mEq} / \mathrm{L}$ & 1,9 & 1,2 & 2,2 & 1,9 & NS \\
\hline$\%$ Potasio $<5,5 \mathrm{mEq} / \mathrm{L}$ & 59,3 & 33,8 & 55,9 & 42,2 & NS \\
\hline$\%$ Potasio $\geq 5,5 \mathrm{mEq} / \mathrm{L}$ & 40,7 & 33,8 & 44,1 & 42,2 & NS \\
\hline Albúmina $(g / d l)$ & 3,6 & 0,2 & 3,6 & 0,3 & NS \\
\hline$\%$ albúmina $\geq 3,5$ g/dl & 76,8 & 34,8 & 70,6 & 42,3 & NS \\
\hline Proteínas (g/dl) & 6,2 & 0,4 & 6,4 & 0,6 & NS \\
\hline$\%$ proteínas $\geq 6 \mathrm{~g} / \mathrm{dl}$ & 72,2 & 40,52 & 76,5 & 40,4 & NS \\
\hline Fósforo (mg/dl) & 4,3 & 1,1 & 5,4 & 1,3 & 0,041 \\
\hline$\%$ fósforo $<4,5 \mathrm{mg} / \mathrm{dl}$ & 56,1 & 41,2 & 29,4 & 36,6 & 0,055 \\
\hline$\%$ fósforo $\geq 4,5 \mathrm{mg} / \mathrm{dl}$ & 44 & 41,2 & 70,6 & 36,6 & 0,055 \\
\hline Calcio (mg/dl) & 8,7 & 0,8 & 8,6 & 0,8 & NS \\
\hline \% calcio $8,4-9,5 \mathrm{mg} / \mathrm{dl}$ & 51,8 & 38,4 & 51,8 & 45,3 & NS \\
\hline
\end{tabular}

AeS: Alfabetización en Salud, GPI: ganancia de peso interdiálisis, DE: desviación típica.

\section{Discusión}

Los datos alcanzados en nuestro estudio revelan un bajo nivel de AeS de nuestros pacientes, similares a los resultados obtenidos en otras muestras de población ${ }^{10,11}$, lo que dificulta el entendimiento, la comprensión e interpretación de la información relacionada con su estado de su salud y los cuidados que puedan precisar. Estudios previos $^{4,12}$ ya objetivaron asociaciones entre el nivel de autocuidados deficientes y un bajo nivel de AeS, debido a la falta de comprensión de las informaciones que los profesionales sanitarios proporcionamos acerca del estado de salud de los pacientes.

La HD es una terapia que conlleva varias adaptaciones importantes del estilo de vida del paciente ${ }^{1}$, que implica la realización de una serie de autocuidados y requiere la adherencia a un régimen terapéutico, incluyendo restricciones dietéticas, restricciones en la ingesta de líquidos y toma de fármacos de forma regular ${ }^{13}$. Los pacientes con AeS limitada tienen dificultad para realizar tareas de autocuidados para las enfermedades crónicas ${ }^{14}$. En nuestro estudio, aquellos pacientes con un nivel de AeS limitada presentaron menor tasa de cumplimiento terapéutico. Este mismo comportamiento se objetiva en la publicación de Green y $\operatorname{cols}^{14}$, en la que pacientes con AeS limitada tienen menos capacidades para seguir las restricciones dietéticas y de ingesta de líquidos prescritas, incluso objetivan un $0,6 \%$ de sesiones perdidas frente a $0,3 \%$ en el grupo de pacientes con AeS adecuada, aunque este parámetro no se analizó en nuestra muestra.

Jia y cols. en su artículo ${ }^{15}$ refieren que la restricción hídrica es el aspecto del tratamiento más difícil de cumplir, siendo necesarios conocimientos y habilidades adecuadas para implementar dicha recomendación. En nuestra muestra la ingesta hídrica no es un parámetro con alto grado de IT, únicamente un $28,36 \%$. Sin embargo, Mellon y col. ${ }^{13}$ en su muestra determinan que el grado de IT para la ingesta hídrica es del $62 \%$. La diferencia puede estar en el punto de corte, donde han establecido $2000 \mathrm{~g}$, mientras que en nuestro estudio se ha aumentado esa cifra hasta los $2300 \mathrm{~g}$, ya que hemos tenido en cuenta la GIP en los periodos de fin de semana que en nuestra Unidad se ha establecido en 2500 g. Lo mismo ocurre con el fósforo. El grado de IT en el estudio de referencia ${ }^{13}$ es del $28 \%$ mientras que, en 
nuestra muestra, alcanza un 57\%. Sin embargo, la unidad de medida empleada para el fósforo es diferente, lo que puede explicar la diferencia de cumplimiento entre ambos estudios.

En nuestro estudio, los pacientes de mayor edad presentan un grado de cumplimiento terapéutico superior al de los pacientes más jóvenes, datos que concuerdan con los obtenidos en otras investigaciones ${ }^{13,16}$. Esto puede explicarse por la dificultad que perciben los pacientes más jóvenes para integrar las restricciones necesarias a su estilo de vida ${ }^{13}$. Una reciente revisión sobre los factores que influyen en la adherencia terapéutica a las restricciones de la dieta en los pacientes en HD, explica este fenómeno por una mayor vida social de los jóvenes (con mayor número de ocasiones para realizar transgresiones); y un menor consumo de sodio en los más mayores (que contribuye a una menor GPI) y mejor manejo de la autoeficacia de los más mayores por tener más experiencia en adaptar su estilo de vida a sus comorbilidades $^{16}$. Sin embargo, una reciente revisión sistemática, sobre AeS en pacientes con enfermedad renal crónica, no encontró relación entre la edad y bajos niveles de $\mathrm{AeS}^{17}$. Esta revisión indica una prevalencia media de AeS limitada en pacientes en diálisis del 27\% (IC 95\% de 19 a 35\%), muy inferior a la de nuestra muestra (inadecuada $51,43 \%)^{17}$. Estas diferencias pueden deberse a la heterogeneidad de instrumentos utilizados en los distintos estudios. En nuestro caso decidimos utilizar el HLS-EU-Q, por estar validado en población española y haber sido utilizado por otros estudios en pacientes crónicos de nuestro país. La revisión de Taylor y cols achaca esta heterogeneidad al uso de versiones abreviadas, que cuando se comparan prevalencias entre sí, no se detecta esta heterogeneidad ${ }^{17}$.

El parámetro con mayor grado de IT en nuestra muestra es el fósforo sérico presente casi en un $57 \%$ de los casos, cifra superior a la que refiere la bibliografía ${ }^{18}$. El paciente en HD debe adaptarse a restricciones dietéticas y presentar una buena adherencia tanto al tratamiento farmacológico como a las sesiones dialíticas ${ }^{1}$. La restricción dietética ha sido en el abordaje terapéutico clásico de la hiperfosfatemia, aconsejándose una restricción del aporte alimentario de fósforo a 800$1000 \mathrm{mg} / \mathrm{di}^{19}{ }^{19}$. Sin embargo, el cálculo de esta dosis diaria en muchos casos es dificultosa, y en mayor medida con los hábitos alimenticios actuales, con el abuso de alimentos procesados, con un alto contenido en fósforo inorgánico (con una alta tasa de absorción intestinal); y las dificultades que comporta un inadecuado etiquetado y la falta de cultura en nuestro entorno de consultar los mismos $^{20-22}$. Millazi y cols ${ }^{18}$ en su revisión sistemática demuestran que las intervenciones educativas y conductuales redujeron de forma significativa los niveles de fósforo sérico en una media de $-0,23 \mathrm{mmol} / \mathrm{L}$.

La falta de conocimientos por parte del paciente dificulta que éstos comprendan la importancia de adherirse a dichos regímenes de tratamiento, de ahí, que el fomento de la AeS sea un objetivo cada vez más frecuente para los pacientes con Enfermedad Renal Crónica ${ }^{5}$, ya que las personas con bajos niveles de AeS presentan peores indicadores de salud. Si el nivel de AeS no es adecuado, los pacientes carecerán de las habilidades y los conocimientos necesarios, lo que evitará su plena participación en la toma de decisiones ${ }^{7}$.

Como limitaciones del estudio, comentar que una variable que no se ha tenido en cuenta en nuestro estudio es la valoración del estado de ansiedad y depresión de los pacientes, variables que pueden influir sobre el cumplimiento o la adherencia terapéutica. Por eso es necesario seguir realizando estudios con muestras mayores $y$ teniendo en cuenta estos aspectos.

No hemos encontrado otros estudios que evalúen la relación entre la adherencia terapéutica medida por resultados clínicos y el nivel de AeS.

Según nuestros datos, concluimos que aquellos pacientes con un mayor nivel de AeS tienen menor grado de IT, con excepción del fósforo, que es el parámetro con menor tasa de adherencia. Además de la AeS, tanto la edad como el sexo, también son factores que influyen en el cumplimiento del régimen terapéutico. Serían necesarios más estudios en poblaciones más amplias, que permitan generalizar o contradecir nuestros resultados. Del mismo modo, se requieren programas educativos que capaciten al paciente a tomar decisiones informadas sobre su autocuidado, mejorando la alfabetización en salud ${ }^{13}$.

Recepción: 16-03-21

Aceptación: 07-04-21

Publicación: 30-06-21 


\section{Bibliografía}

1. Palmer SC, Hanson CS, Craig JC, Strippoli GF, Ruospo M, Campbell K, et al. Dietary and fluid restrictions in CKD: a thematic synthesis of patient views from qualitative studies. Am J Kidney Dis. 2015;65(4):559-73.

2. Guerra VT, Díaz $A E$, Vidal K. La educación como estrategia para mejorar la adherencia de los pacientes en terapia dialítica. Rev Cubana Enfermer 2010;26(2):52-62.

3. Ruiz E, Latorre LI, Delgado A, Crespo R, Sánchez $\mathrm{JL}$. Adherencia al tratamiento farmacológico en pacientes en hemodiálisis. Enferm Nefrol. 2016;19(3):232-41.

4. Salvadó-Hernández C, Cosculluela-Torres $P, B$ BanesMonllor C, Parellada-Esquius N, Méndez-Galeano $\mathrm{C}$, Maroto-Villanova $\mathrm{N}$ et al. Insuficiencia cardíaca en atención primaria: actitudes, conocimientos y autocuidados. Aten Primaria. 2018;50(4):213-21.

5. Costa-Requena G, Moreso F, Cantarell MC, Serón D. Alfabetización en salud y enfermedad renal crónica. Nefrología. 2017;37(2):115-7.

6. Falcón M, Luna A. Alfabetización en salud: concepto y dimensiones. Proyecto europeo de alfabetización en salud. RCyS 2012;2:91-8.

7. Navarro MD, Rudd R, Rosenfeld L, Arrighi E. Alfabetización en salud: implicación en el sistema sanitario. Med Clin. 2016;147(4):171-5.

8. Sorensen $K$, Van den Broucke $S$, Pelikan J, Fullam J, Doyle G, Slonska $S$ et al. Measuring health literacy in populations: iluminating the desing and development precess of the European Health Literacy Survey Questionnarie (HLS-EU-Q). BMC Public Health. 2013;13:1053-58.

9. Kidney Disease: Improving Global Outcomes (KDIGO) CKD-MBD Update Work Group. Kidney Disease: Improving Global Oucomes (KDIG0) 2017. Clinical Practice Guideline Update for de diagnosis, evaluation, prevention and treatment of chronic kidney disease-mineral and bone disorder (CKDMBD). Kidney Int Suppl 2017;7(1):1-59.
10. Young BA. Health literacy in neprhology: why is important? Am J Kidney Dis. 2013;62(1):3-6.

11. Pelayo Alonso $R$, Martínez Álvarez $P$, Merino García S, Labrador Pérez A, Sánchez Cano S, Cobo Sánchez JL. Análisis del nivel de alfabetización en salud en pacientes con insuficiencia renal crónica en hemodiálisis. Enferm Nefrol. 2017;2(3):221-6.

12. Santesmases R, González L, Real J, Borràs A, Sisó $A$, Navarro MD. Alfabetización en salud en pacientes con insuficiencia cardiaca atendidos en atención primaria. Aten Primaria. 2017;49(1):28-34.

13. Mellon L, Regan D, Curtis R. Factors influencing adherence among Irish haemodialysis patients. Patient Educ Couns. 2013;92(1):88-93.

14. Green JA, Mor MK, Shields AM, Sevick MA, Arnold RM, Palevsky PM et al. Associations of health literacy with dialysis adherence and health resource utilization in patients receiving maintenance hemodialysis. Am $\mathrm{J}$ Kidney Dis. 2013;62(1):73-80.

15. Jia S, Huang B, ChuY, LuY, McArthur A. Management of non-adherence to fluid-intake restrictions in hemodialysis patients in a tertiary hospital: a best practice implementation project. JBI Database System Rev Implement Rep. 2016:14(8):309-22.

16. Oquendo LG, Asencio JMM, de Las Nieves CB. Contributing factors for therapeutic diet adherence in patients receiving haemodialysis treatment: an integrative review. J Clin Nurs. 2017;26(2324):3893-905.

17. Taylor DM, Fraser SDS, Bradley JA, Bradley C, Draper $H$, Metcalfe W, et al. A Systematic Review of the Prevalence and Associations of Limited Health Literacy in CKD. Clin J Am Soc Nephrol. 2017;12(7):1070-84.

18. Millazi M, Bonner A, Douglas C. Effectiveness of educational or behavioral interventions on adherence to phosphate control in adults receiving hemodialysis: a systematic review. JBI Database System Rev Implement Rep 2017;15(4):9711010.

19. Noori N, Sims JJ, Kopple JD, Shah A, Colman S, Shinaberger $C S$ et al. Organic and Inorganic Dietary 
Phosphorus and Its Management in Chronic Kidney Disease. Iran J Kidney Dis. 2010;4(2):89-100.

20. Osuna Padilla IA, Leal Escobar G, Garza García $C A$. Manejo nutricional de la hiperfosfatemia en la enfermedad renal crónica. Nutr clín diet hosp 2017; 37(4):140-8.

21. Lou-Arnal LM, Arnaudas-Casanova L, CaverniMuñoz A, Vercet-Tormo A, Caramelo-Gutiérrez R, Munguía-Navarro $P$, et al; Grupo de Investigación ERC Aragón. Hidden sources of phosphorus: presence of phosphorus-containing additives in processed foods. Nefrología 2014;34(4):498-506.

22. Lou-Arnal LM, Caverni-Muñoz A, ArnaudasCasanova L, Vercet-Tormo A, Gimeno-Orna JA, SanzParís $A$ et al. The impact of processing meat and fish products on phosphorus intake in chronic kidney disease patients. Nefrología. 2013;33(6):797-807.

Este artículo se distribuye bajo una Licencia Creative Commons Atribución-NoComercial 4.0 Internacional. https://creativecommons.org/licenses/by-nc/4.0/

Open Access (c) (7) (8) 